\title{
ALGUNOS ASPECTOS DEL MODELO HEMPELIANO DE EXPLICACIÓN HISTÓRICA
}

1. Resulta difícil no encontrar, en las discusiones en torno a la cientificidad de la historia, alguna alusión al modelo de explicación de Hempel. Sobre todo, aquellos que defienden la posibilidad de contar con explicaciones científicas sobre la realidad histórico-social, se ven casi obligados a empezar sus análisis con una crítica de las filosofías de la historia especulativas y terminarlos con una referencia -en algunos casos también crítica - a Hempel. Su modelo de explicación ha sido considerado, en general, como una reconstrucción formal adecuada del procedimiento de explicación científica. Si la historia es cada vez menos ignorada y menos maltratada por los filósofos de la tradición angloamericana, y si se puede pensar en algún tipo de acercamiento entre dicha tradición y la teorfa marxista de la historia, esto se debe en gran medida a Hempel. De ahí la necesidad de volver al modelo hempeliano, analizando sus supuestos filosóficos -implícitos o explícitos--, así como sus principios teóricos básicos, para ver cuáles son los aportes de este filosofo a la ciencia de la historia.

2. El modelo de explicación científica de Hempel y Oppenheim, presentado en su artículo "La lógica de la explicación" (1948), es bien conocido y ya ha sido ampliamente discutido, como también lo ha sido el concepto de esquema explicativo presentado por Hempel en "La función de las leyes generales en la historia" (1942). Además, habría que tomar en cuenta las modificaciones introducidas en dos articulos posteriores: "Explanation in Science and in History" (1962) y "Aspects of Scientific Explanation" (1965). No entraré, pues, en el análisis detallado de este modelo ni en las críticas que se le han hecho. ${ }^{1}$ Me limitaré a señalar algunos de los supuestos fundamentales y algunos de los problemas con los que se enfrenta dicho modelo en el caso de la explicación histórica.

Para que una explicación científica sea correcta, Hempel y Oppenheim establecen que debe cumplir ciertas condiciones, tanto de tipo lógico como de tipo empírico (SLE, pp. 247-248). El siguiente esquema es un resumen de la estructura lógica de la explicación científica, según estos autores (SLE, p. 249):

1 Para una exposición detallada del modelo nomológico-deductivo de explicación histórica, así como de las principales críticas que se le han hecho y las respuestas a estas objeciones, véase J. Graue, La explicación histórica, México, Instituto de Investigaciones Filosóficas de la UNAM, 1976. 


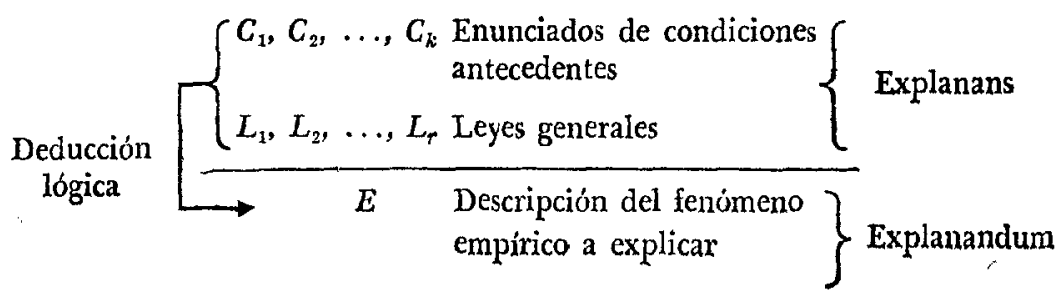

La concepción general de explicación por inclusión deductiva del explanandum o fenómeno a explicar bajo leyes o principios teóricos generales es lo que se llama modelo nomológico-deductivo de explicación (explicación N-D). ${ }^{2}$

La explicación consiste en responder a la pregunta: ‘por qué se afirma el fenómeno del explanandum?, mostrando que éste resultó de ciertas circunstancias particulares $C_{1}, C_{2}, \ldots, C_{k}$, de acuerdo con leyes generales $L_{13}, L_{2}$, $\ldots, L_{r}$. El explanandum es, por lo tanto, una consecuencia lógica del explanans: dadas $C_{1}, G_{2}, \ldots, G_{k}$ y $L_{1}, L_{2}, \ldots, L_{r}$ la ocurrencia del fenómeno aludido en el explanandum era de esperarse.

La inclusión de las condiciones particulares bajo leyes generales es esencial, pues sólo dichas leyes pueden mostrar la relación que existe entre el acontecimiento que se quiere explicar y el conjunto de condiciones particulares, dándoles sentido explicativo. "Una explicación N-D tendrá que contener, en su explanans, algunas leyes generales que se requieren para la deducción del explanandum, es decir, cuya supresión haría el argumento inválido" (ASE, p. 338).

Las explicaciones causales - que consisten en señalar la causa o las causas de un acontecimiento - se conforman al modelo de explicación N-D, en virtud de que suponen implícitamente que hay ciertas leyes generales que relacionan la ocurrencia de un conjunto complejo de circunstancias, consideradas como condiciones suficientes, con la ocurrencia del acontecimiento (efecto) que se quiere explicar.

No toda explicación científica está basada en leyes de forma estrictamente universal. Hempel debe reconocer que hay leyes no universales, de forma probabilistica o estadistica, implicadas en explicaciones científicas. Justamente, las leyes que se utilizan en las explicaciones de las ciencias sociales tienen carácter más bien estadístico, no universal. Hempel destaca que las explicaciones probabilísticas comparten algunas características con las explicaciones nomológico-deductivas. En ambos casos el fenómeno en cuestión se explica por referencia a otros acontecimientos, con los que el explanans está relacionado por medio de leyes. La diferencia radica en que, en el caso de la explicación probabilística, las leyes no son de forma universal, sino de forma estadística o probabilística. Esto significa que, mientras que en la explicación

2 Hempel acepta el término de modelo de "ley inclusiva" sugerido por Dray. Por "ley inclusiva" hay que entender la ley o leyes presupuestas en las explicaciones históricas. Este término daría cuenta, entonces, tanto del modelo nomológico-deductivo como del probabilístico-inductivo. 
N-D el explanandum se deduce con certeza de la información contenida en el explanans, en la explicación probabilística lo más que puede decirse es que el explanans hace al explanandum probable (en cierto grado), y no lo implica con certeza deductiva. Por ello, hay una gran diferencia lógica entre las explicaciones N-D y las explicaciones probabilísticas, cuyos argumentos explicativos son de carácter inductivo (explicación P-I o probabilístico inductiva).

3. Mientras que los filósofos idealistas de la historia (Croce, Collingwood, Oakeshott) sostienen que existe una división tajante entre las ciencias naturales y las "ciencias del espíritu", para Hempel esta división, además de arbitraria, es falsa. En contra de estos filósofos, que defienden el uso de métodos claramente subjetivos tales como la "empatía", la "comprensión", la "hermenéutica", Hempel sostiene la posibilidad de constituir la historia como ciencia. Parte del supuesto que afirma la unidad metodológica de la ciencia, por lo que la historia - si quiere alcanzar la condición de ciencia- deberá satisfacer los postulados del modelo nomológico-deductivo de explicación, o sus variantes propuestas para la historia. Como dijimos, en dicho modelo las leyes cumplen una función teórica indispensable; sin su presencia no se podría hablar de explicación. La tarea de Hempel consistirá, entonces, en mostrar la existencia y tunción de leyes generales en la historia.

Para que una explicación sea completa, el explanandum debe deducirse de leyes generales y de condiciones antecedentes específicas. Hempel menciona varias maneras en las que una determinada explicación puede "desviarse" de lo que él ha caracterizado como modelos básicos de explicación científica. ${ }^{3}$ Puede haber, así, explicaciones elípticas en las que no se mencionan ciertas leyes o hechos particulares porque se les considera obvios; explicaciones parciales en las que no se menciona ninguna ley o hipótesis general en virtud de la cual las condiciones iniciales señaladas explicarian el acontecimiento en cuestión, pero que sin embargo están sugeridas como base de la explicación. Estas explicaciones suprimen, pues, parte del explanans, por lo que se trata de explicaciones incompletas. Las explicaciones probabilísticas también pueden ser "incompletas", pero no por no ser deductivas, ya que se construyen independientemente de la asunción de leyes estrictamente universales, pueden ser parciales en la medida en que confieren probabilidad a enunciados más débiles.

En la literatura historiográfica es muy improbable que una explicación histórica se nos dé con base en hipótesis universales $y$, generalmente, tales explicaciones no incluyen una declaración expresa de las hipótesis o leyes que utilizan. Hempel opina que esto sucede por dos razones: a) se trata de hipótesis que se relacionan ya sea con la psicología individual o social, ya sea con proposiciones conocidas por todos, por lo que los historiadores no se sienten obligados a formularlas explícitamente (hay que señalar, sin embargo,

3 En "Aspects of Scientific Explanation", Hempel habla de tres tipos básicos de la explicación cientifica: nomológico-deductivo, inductivo-estadistico y deductivo-estadístico. En "Explanation in Science and in History" sólo menciona dos: el nomologico-deductivo y el inductivo-probabilístico. 
que no hay un solo acontecimiento histórico que pueda explicarse de esa manera); b) a menudo sería difícil formular tales supuestos con precisión de modo que a la vez concuerden con la experiencia empírica disponible relevante. Términos como "por tanto", "en consecuencia", etcétera, nos muestran que de alguna manera se está haciendo uso de leyes o hipótesis universales para vincular las condiciones iniciales con el acontecimiento que se quiere explicar. El rigor de una explicación se pondría de manifiesto demostrando que estas conexiones no son arbitrarias, es decir, que no se basan en regularidades que no existen, sino en leyes o hipótesis universales.

Aun cuando, en el terreno de la historia, sea posible construir explicaciones no sólo con base en leyes o hipótesis generales, sino también con base en hipótesis de probabilidad, teniendo entonces explicaciones de carácter probabilistico y no causal, "es cierto que en general las condiciones, y especialmente las hipótesis universales implícitas, no se encuentran claramente indicadas y no pueden completarse sin ambigüedad. (En el caso de las hipótesis probabilísticas, por ejemplo, los valores probabilísticos implicados podrán ser conocidos a lo más de manera aproximada.)" (GLH, p. 351).

Las explicaciones históricas no son lo bastante explícitas para ser calificadas como elípticas o parciales, por lo que Hempel sugiere, entonces, que los análisis explicativos de los acontecimientos históricos no ofrecen una explicación en el sentido estricto, sino un esquema o boceto de explicación (explanation sketch). Este esquema consistiria en una indicación más o menos vaga de cuáles son las leyes universales que se requieren para completar el boceto y cuáles son las condiciones iniciales que se consideran necesarias para la explicación de un acontecimiento. El proceso de completar el esquema de explicación necesita, por tanto, tener en cuenta las condiciones relevantes y hacer explícitas las hipótesis universales que se encuentran implícitas en la explicación.

Frente a un acontecimiento histórico cualquiera se pueden tener numerosos esquemas de explicación distintos. Estos esquemas se irían completando en forma creciente, precisándose cada vez más por medio del avance de las investigaciones históricas. La manera de valorar los múltiples bocetos explicativos consistiria en reconstruir primero el argumento que constituye la columna vertebral de los esquemas. Hempel insiste en que hay que poner el acento en los nexos ("de ahi", "por lo tanto", "en consecuencia") en los que el historiador encuentra el paso lógico a la conclusión. Al encontrar los supuestos teóricos que están detrás de esas conexiones, se verán las falacias en las que descansa la investigación, así como las hipótesis universales y probabilísticas que posibilitan una explicación científica.

4. La tesis central sostenida por Hempel es que toda explicación científica implica la subsunción de su objeto de conocimiento bajo regularidades generales. El modelo N-D y el modelo P-I —submodelos del modelo de la ley inclusiva-, y sus diversas variantes, son idealizaciones teóricas que para $\mathrm{Hem}$ pel no pretenden, realmente, describir de qué manera formulan los científicos sus explicaciones. El propósito del modelo de la ley inclusiva es el de "pro- 
porcionar explicaciones, o reconstrucciones racionales, o modelos teóricos, de ciertos modos de explicación científica" (ESH, p. 15). Los modelos presentados tienen, pues, un carácter muy vago, por lo que no son -ni pretenden ser- un análisis del complejo proceso de producción del conocimiento.

Los "esquemas explicativos" con los que Hempel pretende dar cuenta de la manera como explican los historiadores, son todavía más vagos. En su primera formulación, están calcados del modelo más fuerte presentado por Hempel (el modelo N-D): en las explicaciones históricas, los acontecimientos históricos deben deducirse, en principio, de leyes generales.

El modelo hempeliano de explicación histórica tiene un mérito innega: ble: afirmar la posibilidad y necesidad de constituir la historia como ciencia. Esto supone el abandono de la mera crónica, es decir, la historia como la simple presentación en orden cronológico de una serie de acontecimientos, que no rebasa el plano descriptivo. Bastaría mencionar las múltiples "historias" nacionales y universales escritas, sin contar con las "historias" de la filosotía, de la literatura, de la ciencia, del arte, para comprobar que se trata de meras descripciones no explicativas del proceso histórico.

Hay que rescatar, pues, del modelo hempeliano, la idea de que la historia tiene como fin explicar y que, por tanto, los historiadores no pueden conformarse con la presentación de un conjunto de hechos arreglados cronológicamente, sino que deben mostrar, de manera no arbitraria, las conexiones entre ellos. Puede discutirse, sin embargo, si dichas conexiones tienen que expresarse exclusivamente -como lo supone Hempel — en forma de leyes veriticables empíricamente, y si es posible encontrar explicaciones históricas que se ajusten de manera estricta al modelo nomológico-deductivo.

En lo que respecta a esto último se estaria criticando, no tanto el modelo en sí, como los límites de su aplicación. Podría aceptarse como hipótesis que el modelo proporciona una reconstrucción formal correcta de las explicaciones en las ciencias naturales, pero al aplicarlo al campo de la historia, pretendiendo que los acontecimientos históricos que se busca explicar deben deducirse de leyes generales, resulta un fracaso.

Los ejemplos de explicación histórica que Hempel da en su primer artículo son muy sospechosos: rara vez -o nunca - se refieren a acontecimientos propiamente históricos. La posibilidad de construir una explicación tan "limpia" desde el punto de vista lógico sobre algún acontecimiento realmente relevante para la ciencia de la historia, es muy dudosa. Posteriormente introduce ejemplos que, aunque tienen más que ver con la psicología, se acercan más al tipo de acontecimientos estudiados por la ciencia social. Del análisis de estos ejemplos se desprende que, para Hempel, si bien las explicaciones históricas siguen siendo nomológicas, ya no rige el requisito de la inclusión de al menos una ley de carácter universal en la explicación. Las explicaciones históricas se basan más o menos explícitamente en generalizaciones de carácter ampliamente probabilistico, que expresan, no uniformidades estrictas, sino más bien tendencias (ESH, p. 19). Sin embargo, no queda nada claro qué es lo que Hempel entiende por "tendencia"; si tendencia es algo distinto a una ley probabilística - la cual puede ser muy especifica-, entonces habría que 
determinar qué tan general es una tendencia y plantear en otros términos el problema de la verificación.

Para Hempel, el uso de conceptos como dependencia, determinación, en la ciencia de la historia, trae implicita la referencia a algún tipo de principio teórico general. Pero, en virtud de que dichas generalizaciones que sustentan las explicaciones rara vez se especifican de manera explícita, las explicaciones históricas son incompletas —son "esquemas de explicación". Por la vaguedad y amplitud de dichos "esquemas" no es posible determinar con precisión cómo deberían completarse las explicaciones históricas. A la pregunta de cuáles serían las leyes específicamente históricas, Hempel responde únicamente que los historiadores "toman" las hipótesis universales que utilizan en sus explicaciones de otros campos de la investigación cientffica. Dichas hipótesis proceden de generalizaciones precientíficas de experiencias cotidianas; las explicaciones históricas también pueden apoyarse en leyes generales de otras ciencias ya constituidas, como la f́́sica o la química. Para Hempel, "es injustificable y fútil intentar la demarcación de fronteras precisas entre los diferentes campos de la investigación cientifica y un desarrollo autónomo de cada uno de estos campos" (GLH, p. 356). Cabe preguntarse si la fuerza explicativa de la historia puede hacerse depender de "generalizaciones precientíficas", generalmente obvias y de carácter dudoso, y si los historiadores pueden tomar prestadas leyes de otras disciplinas utilizándolas, sin más, en la explicación de los acontecimientos históricos.

5. La condición empirista del modelo hempeliano es lo que, sin embargo, resulta más cuestionable, sobre todo por pretender que las explicaciones históricas deben conformarse a las explicaciones del tipo de ley inclusiva. El problema estaría en si es posible encontrar en la historia leyes o regularidades del tipo exigido por dicho modelo de explicación. Si por "ley" se entiende una universalidad estricta y además verificable empíricamente, será difícil que encontremos este tipo de leyes en la historia. No debe confundirse el intento de los historiadores por establecer conexiones concretas, no arbitrarias - -es decir, respaldadas teóricamente-, con el intento de formular leyes. Los historiadores pueden establecer los mecanismos que subyacen a los acontecimien. tos históricos, o descubrir su causa o sus causas, sin necesidad de formular leyes de la historia, de forma condicional universal, verificables por medio de la experiencia.

Un supuesto generalmente aceptado por el empirismo es aquel según el cual se puede conocer directamente cualquier cosa - fenómeno o ente subyacente- por medio de la observación, sin asunciones teóricas previas. Lo básico de la actividad científica es, por tanto, la observación y, a partir de ella, se procede a aumentar el grado de certeza de nuestros conocimientos. El objetivo cientifico principal es el de probar la verdad de los enunciados que se consideran como conocimiento. La realidad sería, para el empirista, un conjunto más o menos complejo de hechos que son directamente observables, es decir, observables independientemente de cualquier teoría. Las proposiciones acerca de hechos inobservables tendrían que ser traducibles, en principio, a 
proposiciones observacionales, mediante criterios muy estrictos, pues en caso contrario esas proposiciones sólo serian un instrumento heurístico para ordenar la observación. En general, la tradición empirista, al no trazar la distinción entre lo observable y lo inobservable, se compromete con la aceptación del mundo tal y como aparece, con lo que sus análisis se convierten en simples descripciones, eliminando la posibilidad de cualquier explicación.

Hempel no es un empirista radical; en su posición encontramos cambios relevantes que lo alejan de las tesis generalmente sostenidas por esa tradición. Hempel acepta que el objetivo de una teoría es proporcionar una comprensión más profunda y exacta de las regularidades que revelan la observación directa o experimental de una clase de fenómenos. Para ello, la teoría no se queda en lo observable de esos fenómenos sino que los interpreta como manifestaciones de entidades y procesos que estarian detrás o debajo de ellos. La teoría formula, entonces, leyes teóricas o principios teóricos que estarían gobernando dichas entidades y procesos subyacentes. "La investigación científica - escribe Hempel-, y en especial la explicación teórica, persigue un tipo de objetivo de penetración en los fenómenos que se alcanza mediante una unificación sistemática, mediante la mostración de los fenómenos como manifestaciones de estructuras y procesos subyacentes comunes que se ajustan a principios básicos específicos contrastables" (FCN, p. 125).

Las construcciones teóricas dan cuenta, entonces, de las regularidades de los fenómenos, ocultas a la observación directa. Para Hempel no se requiere llegar a los enunciados teóricos por vía de generalizaciones - de enunciados que se refieren a observaciones directas - sino que los considera como hipótesis, siempre y cuando éstas sean susceptibles de contrastarse con la experiencia. La teoría nos permitiría, así, ir conociendo cada vez más las cosas acerca de la realidad.

Sin embargo, a pesar de la aceptación, en Hempel, de la importancia del papel que juega la teoría en el conocimiento científico, en sus análisis sobre la explicación histórica ya no se habla de una teoría sobre la que estaría montada la explicación. Considero central la tesis de que toda explicación se encuentra necesariamente subordinada a una teoría. Sin teoria, es decir, sin el conjunto de principios y conceptos que definen teóricamente el objeto que se quiere conocer, la discusión sobre cuáles serían las explicaciones realmente científicas se vuelve estéril. Plantear el problema de la explicación histórica fuera de la delimitación del objeto de la ciencia de la historia, es decir, al margen del campo en el que esa ciencia ha de producir conocimiento, no parece llevar a ninguna parte. La determinación de si la explicación sobre un acontecimiento histórico es o no científica, no puede darse independientemente del problema de la teoría que sostiene tal explicación: sólo en ese nivel es posible plantear la cuestión de la cientificidad de las explicaciones.

Como cualquier otra ciencia, la historia tiene que construir su teoría: sólo mediante un sistema de conceptos teóricos que fundamente los métodos y prácticas de los historiadores, y que defina el objeto que se quiere conocer, la historia puede llegar a constituirse como ciencia. La definición teórica específica de lo que se quiere estudiar es algo de lo que la historia no puede 
prescindir. Al no tomar en cuenta esta exigencia, Hempel pasa por alto, entre otras cosas, el problema del reconocimiento científico de los hechos históricos. En tanto que en el trabajo de todos los historiadores está implicito el funcionamiento de una "teoría" que guía los criterios de selección de "hechos" y de interpretación de los mismos, sin una definición teórica de lo que es la historia no hay conocimiento de la historia, no hay posibilidad de explicar los objetos "históricos".

Por otra parte, si Hempel acepta que "la ciencia plantea la pregunta '¿por qué?' también en relación con las uniformidades expresadas por tales leyes (leyes fácticas), y trecuentemente la responde básicamente de la misma manera, a saber, subsumiendo las uniformidades bajo leyes más inclusivas, y eventualmente bajo teorias comprensivas" (ESH, p. 11), tendría que reconocer que también en la ciencia de la historia, como en cualquier otra explicación científica, intervienen leyes de muy distintos niveles: desde principios muy generales, no contrastables directamente - principios que tienen como fin organizar el campo teórico, tratando de definir cuáles son los conceptos base que se van a utilizar-, hasta leyes fácticas como, por ejemplo, las leyes económicas que se pueden comprobar de manera directa. No es suficiente, pues, decir que las explicaciones que se fundamentan en principios muy generales son erróneas o no válidas, sino que habría que determinar en qué nivel de abstracción se mueve dicha explicación.

Por ejemplo, Hempel señala que hay algunos tipos de presupuestos que, sin ser hipótesis universales, se encontrarían implicados en las conexiones entre el explanandum y el explanans en las explicaciones históricas. Nos dice que pretender que del examen de la situación económica pueden desprenderse explicaciones de otros fenómenos sociales (religión, arte, ciencia, etcétera), o absolutizar un factor y, a partir de ello, pretender una explicación total de otros factores, son algunos de los presupuestos erróneos tomados a veces como hipótesis universales. Eso tiene como consecuencia el que la explicación no sea válida.

Supongamos, por un momento, que Hempel aludiera, como presupuesto erróneo, al principio marxista de la determinación en última instancia por la base cronológica, 0 al principio según el cual la lucha de clases es el motor de la historia, efectivamente mencionado por él. En ese caso debería, paralelamente, presentar, dentro de su modelo de explicación, ejemplos físicos del mismo nivel de abstracción que aquéllos, cosa que no hace. Por otra parte, si así lo hiciera se encontraría con todas las dificultades con que tropezó el modelo neopositivista, piramidal, de la ciencia; y en particular con el de los conceptos y entes teóricos. Parecería, pues, una crítica excesiva considerar erróneos aquellos presupuestos sin contar siquiera con un análisis de presupuestos del mismo nivel de abstracción en una ciencia tan constituida como la física. Lo que resulta impracticable para la física no puede razonablemente exigirse para la ciencia de la historia.

6. No se trata de disminuir el alcance y el valor de la contribución de Hempel. Por el contrario, he intentado señalar aquello que puede ser recu- 
perable de sus tesis sobre cuáles son las condiciones objetivas con las que una explicación debe cumplir para ser auténtica, para ser realmente científica, en contraposición a las características de las explicaciones espurias o seudoexplicaciones. La aportación de Hempel a la teoría de la historia reside, pues, en su interés por establecer las condiciones formales mínimas de las explicaciones históricas. Pero la necesidad de no conformarnos con descripciones, y mucho menos con interpretaciones ideológicas de los acontecimientos históricos -pasados y sobre todo presentes_, exige la existencia de una teoría objetiva y con fuerza explicativa, que sea una verdadera alternativa al subjetivismo y la arbitrariedad, y esto es mucho más de lo que Hempel propone. Por ello, me he limitado a señalar algunos supuestos generales - muchas veces no declarados- sobre los que descansan las tesis defendidas por este autor, así como su verdadero alcance.

El desconocimiento de la especificidad del objeto teórico de la ciencia de la historia, y de cada una de las prácticas científicas, la ausencia de una concepción explícita del todo social, en suma, la ausencia de una teoría de la historia, constituye un grave obstáculo en la determinación hempeliana de la especificidad de la lógica de las explicaciones históricas. Justamente porque esta teoría es la que determina, no sólo qué leyes hay - cosa que Hempel no pone en cuestión, puesto que sólo afirma que debe haber leyes-, sino si las leyes son o no necesarias en una explicación, el modelo --por más ventajas que posean su simplicidad y su concisión - aparece como totalmente insuficiente.

Para Hempel, ubicado dentro de la tradición del empirismo lógico, la ciencia debe ajustarse a ciertos requisitos lógicos, algunos de los cuales son enunciados en su modelo nomológico-deductivo. Asi, por ejemplo, las leyes deben tener forma de generalizaciones universales, o la relación entre explanandum y explanans debe ser deductiva o cuasi deductiva. La elección entre teorías alternativas dependería de que se ajustaran, como requisito mínimo, a estas condiciones formales. En el caso particular de las explicaciones, he querido subrayar la tesis según la cual la naturaleza de la explicación depende de la naturaleza de la teoría, cuestionando cualquier intento por legislar a priori las formas de la explicación. Las condiciones formales no pueden usarse, por lo tanto, como criterios para elegir entre teorías alternativas, ya que sería la propia teoría la que determina qué condiciones formales son posibles y necesarias. Por ejemplo, las leyes universales pueden no tener la forma de generalizaciones universales, en virtud de que la necesidad histórica es tendencial y no de una regularidad estricta; la relación entre los principios más generales y los análisis concretos puede no ser deductiva; el tipo de causalidad puede no ser lineal, en virtud del carácter sobredeterminado de los hechos sociohistóricos. Sólo desde la teoría de la historia será posible determinar si es o no posible realizar análisis concretos que no necesariamente tengan que hacerse por vía de leyes universales. Sólo desde allí puede determinarse si las pretensiones de relevancia, tanto de las condiciones iniciales como de las leyes o principios generales, son o no espurias, y cuán fuerte o 
cuán débil es la base que nos permite explicar la ocurrencia de un acontecimiento. ${ }^{4}$

Instituto de INVESTigaciones Filosóficas

Corina de YTURBe

Universidad Nacional Autónoma de MÉxico

\section{BIBLIOGRAFfA}

C. G. Hempel, "The Function of General Laws in History", en P. Gardiner (ed.), Theories of History, N. Y., The Free Press, 1959. (abrev. GLH)

-, "Explanation in Science and in History", en R. G. Colodny (ed.), Frontiers of Science and Philosophy, University of Pittsburgh Press, 1962. (abrev. ESH)

—_, "Studies in the Logic of Explanation", en C. G. Hempel, Aspects of Scientific Explanation and other essays, N. Y., The Free Press, 1966. (abrev. SLE)

$\longrightarrow$, "Aspects of Scientific Explanation", en C. G. Hempel, Aspects of Scientific Explanation and other essays, ed. cit. (abrev. ASE)

\footnotetext{
4 Este trabajo forma parte de una investigación más amplia, en la que buscaba comparar tres propuestas para la explicación de la historia: el modelo hempeliano nomológico-deductivo, el modelo intencionalista de von Wright y la teoria marxista de la historia. Pero mientras que la discusión sobre la explicación histórica, tanto en Hempel como en von Wright se da al margen de una teorfa propiamente dicha de la historia, en Marx este tema supone una teoría de la historia, por lo que es imposible reducir su obra a un esquema; así, la comparación resultaba no sólo difícil, sino casi imposible, pero de cualquier modo necesaria en vista del tradicional aislamiento entre la filosofía tradicional y la filosofia marxista. A pesar, pues, de que tanto Hempel como von Wright llegan a plantear problemas reales de una teoría de la historia, y en algunos casos apuntan soluciones a dichos problemas, conducen, de todas maneras, a callejones sin salida para la teoría de la historia.
} 